\title{
Reliability of Envelope Inversion for the High-Frequency Radiation Source Process Using Strong Motion Data: Example of the 1995 Hyogoken Nanbu Earthquake
}

\author{
by Jorge Aguirre and Kojiro Irikura
}

\begin{abstract}
This article presents an application of a procedure to invert the highfrequency radiation process at the source during the 1995 Hyogo-ken Nanbu earthquake using the envelopes of acceleration waveforms from 16 stations. The inversion uses genetic algorithms that compare observed ground motions with synthetic ones calculated using empirical Green's functions. Before the inversion, the reliability of the solutions for models with different grid sizes is checked. It is found that the resolution of the high-frequency radiation is strongly dependent on the number of reliable data and, for this case, it is shown that a coarse grid model with 60 parameters provides reliable results. With use of this model, the inversion of the high-frequency radiation distribution for the 1995 Hyogo-ken Nanbu earthquake was performed, and results showed four distinct zones of high-frequency radiation. The first zone is located in subfault 1 near a step-over from subfault 1 to the Nojima fault and the starting point of the rupture. The second zone is located in subfault 2 near the lower limit of the slip inverted from low-frequency data by many authors. The third and fourth zones are located in subfault 3 , one of them coinciding with the bifurcation of the fault plane into two fault planes (Sekiguchi et al., 2000).

It is concluded that the procedure applied in this article successfully inverts the high-frequency radiation distribution for the 1995 Hyogo-ken Nanbu earthquake. Resolution is restricted to the coarse grid model with 60 parameters because of the limited number of stations, according to the resolution test. The results of the resolution test done in this work can not be generalized; however, they show that it is very important to check the resolution before doing this kind of inversion.
\end{abstract}

Introduction

The source-rupture process is one of the most complex and unknown factors in the characteristics of an earthquake event. Some knowledge has been obtained through the inversion of data in the low-frequency ranges usually less than $1 \mathrm{~Hz}$ (velocity seismograms, accelerograms, Global Positioning System measurements, and interferometer images among others). On the basis of laboratory tests some authors have found friction laws (Dietrich, 1979; Rice and Ruina, 1983) that attempt to explain relations between kinematic and dynamic parameters. The conditions for stress and strength can be measured and controlled in the laboratory, but in actual cases the stress distribution before and after an earthquake and the spatial distribution of strength properties in the fault plane are unknown. The strength of the material for each part of the fault is also unknown. Those properties are impossible to measure directly with the existing technology.
The dynamics of the source process has been studied using the results of kinematic inversions combined with crack modeling to obtain the stress drop, strength-excess distributions, rise-time distribution, and dimensionless stress ratio $S$ (Fukuyama and Mikumo, 1993). The kinematic models are based on the inversion of waveforms with low-frequency contents $(<1 \mathrm{~Hz})$. Because the dynamic process is linked to high-frequency motions more than low-frequency motions, it is desirable to obtain the dynamic description of the source process independently of the results estimated from lowfrequency motions. Because the high-frequency contents of the acceleration waveforms are highly oscillating and incoherent, inversion by fitting synthetic waveforms to observed data is not possible. Instead, several authors have used the envelopes of the accelerograms to study the high-frequency radiation from the source (Gusev and Pavlov, 1991; Cocco and Boatwright, 1993; Zeng et al., 1993; Kakehi and Irikura, 
1996). We use a similar approach here to invert the highfrequency seismograms from the 1995 Hyogo-ken Nanbu earthquake. Contrary to the approach of using Green's functions calculated theoretically (Zeng et al., 1993), we use records of aftershocks as empirical Green's functions (Kakehi and Irikura, 1996). We use Genetic Algorithms (GAs) as a method of inversion instead of the linearized inversion used by Kakehi et al. (1996). An important problem that has not been considered previously is the high-frequency radiation resolution. Here, we check the high-frequency radiation resolution using synthetic high-frequency distributions of different grid-size models. In this resolution test we used the synthetic data at the same 16 stations used for the inversion of the observed data. We conclude that our resolution is restricted to the available data set. The use of an excessive number of parameters leads to a large uncertainty in the solution. For the example of the 1997 Hyogoken Nanbu earthquake inversion, the resolution test shows that resolution is guaranteed with the coarse grid model involving 60 parameters.

\section{Data}

The data records were selected considering that the empirical Green's function method requires at least two records at the same station, one from the mainshock and another from an aftershock or foreshock. For the stations located close to the fault we required records from two aftershocks. Using this criterion we selected a data set of 16 stations, eight of which were operated by the Committee of Earthquake Observation and Research in the Kansai Area (CEORKA) and eight stations operated by the Japan Meterological Agency (JMA). We computed first the ground motions using the empirical Green's function method, then constructed the envelopes with a root-mean-square running window to fit the observed ones. Because of the limited number of available records, we used records of different aftershocks as empirical Green's functions, depending on the stations. Except for the OKA station, we used two aftershocks to simulate the mainshock: one for the Nojima fault (subfault 2; Fig. 1) and the other for the Suma and SuwayamaGousukebashi faults (subfaults 1 and 3, respectively; Fig. 1). The stations and aftershocks used as empirical Green's functions for the inversion are listed in Table 1; the location of the stations is depicted in Figure 2. The epicentral location of the aftershocks is shown in Figure 1. Because the source area of two of the used aftershocks is about $1 \mathrm{~km}^{2}$, the records of the other three aftershocks were corrected by scaling them (constant factor) to produce the same high-frequency spectral level, matching the $\omega^{-2}$ source spectra with constant stress drop.

\section{Method}

We invert the distribution of weights and perturbation times in the source by fitting the synthetic acceleration envelopes to the observed ones. To compute the synthetic ac- celerograms a modified version of the empirical Green's function method (Irikura, 1986) is used. The perturbation times and weights are included in this modified version, for which an explanation follows. According to the scaling law, the number of small events $\left(N^{3}\right)$ with moment $m_{0}$ required to simulate a large event with moment $M_{0}$ is given by

$$
\frac{U_{0}}{u_{0}}=\frac{M_{0}}{m_{0}}=c N^{3}
$$

where $U_{0}$ and $u_{0}$ are the flat levels of the displacement spectra at low frequencies for the large and small events, respectively; and $c$ is a constant introduced to account for the difference in stress drop between the small $\left(\Delta \sigma_{\mathrm{S}}\right)$ and large $\left(\Delta \sigma_{\mathrm{L}}\right)$ earthquakes,

$$
c=\frac{\Delta \sigma_{\mathrm{L}}}{\Delta \sigma_{\mathrm{S}}}
$$

If the Fourier spectra follow the $\omega^{-2}$ model, equation (1) reduces for high frequencies to

$$
\frac{A_{0}}{a_{0}}=c N
$$

where $A_{0}$ and $a_{0}$ are the flat level of the acceleration spectra at high frequencies for the big and small events, respectively. Following the formulation of Irikura (1986), the large-event acceleration waveform can be synthesized using

$$
A(t)=c \sum_{i=1}^{N X} \sum_{j=1}^{N W} \frac{r}{r_{i j}} F_{i j}(t)^{*} a(t)
$$

where $F_{i j}(t)$ is given by

$$
\begin{aligned}
F_{i j}(t)=\delta( & \left.-t_{i j}\right) \\
& +\frac{1}{n^{\prime}} \sum_{k=1}^{(N T-1) n^{\prime}} \delta\left(t-t_{i j}-\frac{(k-1) \tau_{\mathrm{L}}}{(N T-1) n^{\prime}}\right)
\end{aligned}
$$

and

$$
t_{i j}=\frac{\left(r_{i j}-r_{0}\right)}{\beta}+\frac{\xi_{i j}}{V_{r}}
$$

in which the $N^{3}$ has been treated separately $\left(N^{3}=\right.$ $N X \cdot N W \cdot N T$ ) with $i, j$, and $k$ for the number of subfault in the strike direction, width direction, and time, respectively, as shown in the Figure $3 ; \tau_{\mathrm{L}}$ is the rise time of the large earthquake; $\beta$ and $V_{r}$ are the $S$-wave and rupture velocities, respectively.

This formulation is for the homogeneous rupture. For the inhomogeneous rupture, we introduced weight $\left(w_{i j}\right)$ and time perturbations $\left(p t_{i j}\right)$ to account for the irregular distri- 


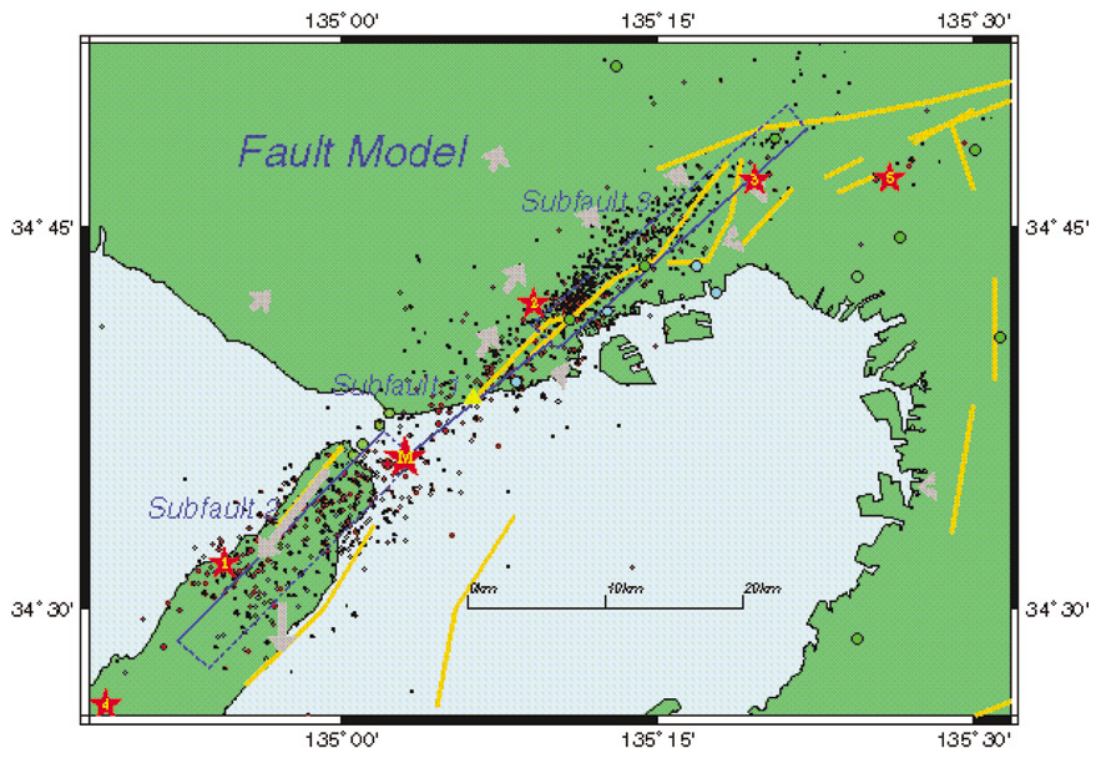

Figure 1. Positions of the subfaults and epicentral locations of the mainshock and aftershocks used in this study. Large star with an $M$ shows the epicenter location of the mainshock. The small stars with numbers inside show the epicentral locations of the five aftershocks used in this study. The traces of the faults are indicated by continuous lines, and the dashed lines show the projection of the other vertices of the fault planes. The dots show the epicentral locations of the aftershocks.

Table 1

Aftershocks Used as Empirical Green's Functions

\begin{tabular}{lcc}
\hline Station & $\begin{array}{c}\text { Aftershock for } \\
\text { subfault 2 (Nojima) }\end{array}$ & $\begin{array}{c}\text { Aftershock for } \\
\text { subfaults 1 and 3 }\end{array}$ \\
\hline $\begin{array}{c}\text { ABE AMG CHY KBU } \\
\text { KMT MKT TDO YAE }\end{array}$ & 23 January, 6:02 & 2 February, 16:19 \\
$\begin{array}{c}\text { AIO AWA HEG KOY } \\
\text { MIN MON WAC }\end{array}$ & 18 February, 21:36 & 25 January, 23:15 \\
OKA & 17 January, 7:37 & 17 January, 7:37 \\
\hline
\end{tabular}

bution of the radiation and for the changes in rupture velocity. Thus, equations (4) and (6) become

$$
A(t)=c \sum_{i=1}^{N X} \sum_{j=1}^{N W} w_{i j} \frac{r}{r_{i j}} F_{i j}(t)^{*} a(t)
$$

and

$$
t_{i j}=\frac{\left(r_{i j}-r_{0}\right)}{\beta}+\frac{\xi_{i j}}{V_{r}}+p t_{i j}
$$

The comparison of the synthetic and observed acceleration records was done using envelopes, not the waveforms. The original signals were filtered between 1 and $10 \mathrm{~Hz}$. The envelopes were calculated using a 2 -sec width root-meansquare running-window from the beginning of the $S$-wave arrival.

\section{Genetic Algorithms}

The inversion is performed by using the GA. The idea of using this analogy of natural evolution to obtain an optimal solution was first proposed by Holland (1975). Thereafter, many variations and combinations were developed to

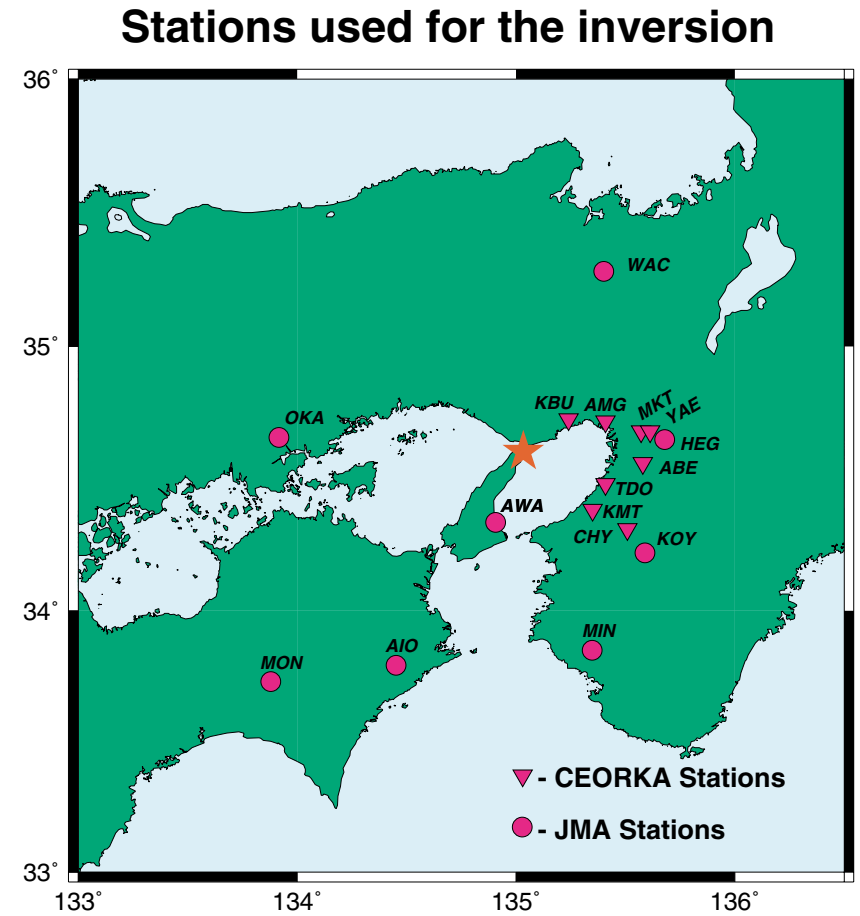

Figure 2. Location of the stations used for the inversion. Filled circles represent the JMA stations. Inverted triangles represent the stations operated by CEORKA. The epicenter of the mainshock is indicated by a star.

improve the speed and performance of the algorithm. The basic GA presumes that the potential solution of any problem is an individual, represented by a set of parameters. The parameters are regarded as the genes. A fitness value is assigned to the individual to evaluate its goodness of fit. This individual competes with many other individuals of the same generation and those who have better fitness value will have higher reproduction probability. After the individuals are 


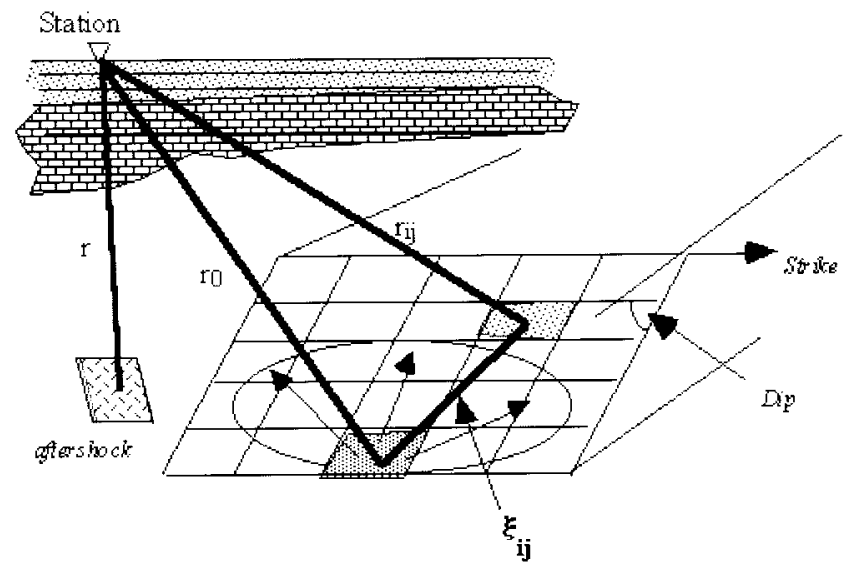

Figure 3. Schematic representation of the empirical Green's function method, where $r, r_{0}$, and $r_{i j}$ are the hypocentral distance of the aftershock, mainshock, and the $i j$ element to the station, respectively. The distance between the $i j$ element and the hypocenter of the mainshock is denoted by $\xi_{i j}$.

selected for the next generation, the crossover operation is applied. The crossover operation works as an interchange of genes between couples of individuals. These combinations may produce better individuals. An additional operation is the mutation. This operation consists of randomly replacing some genes of some individuals selected randomly. These operations help to reduce the probability of being trapped in a local minimum solution. In our particular case, we used a strategy, known as elitist, in which $30 \%$ of the best individuals are copied to the next generation and no crossover operation is applied to them. We use the PGAPack, a parallel GALibrary created by Levine (1996). In the inversion, the genes corresponded to the weights $\left(w_{i j}\right)$ and the perturbation times $\left(p t_{i j}\right)$. Each individual consists of a complete set of weights and perturbation times. The goodness of each individual is evaluated by the sum of the residual values (i.e., difference between the synthetic and the observed envelopes) for the three components of all the stations.

\section{Test of Resolution}

Because a clear relation does not exist $a$ priori between the number of stations used and the number of parameters that can be resolved with confidence from the inversion, a test was performed. For other inversion techniques, smooth constraints provide enough stability of the results. However, that condition can not be introduced to GA inversion. This resolution test was done to find the number of parameters that we may be able to resolve with a certain degree of confidence.

The test was performed using the same number of stations and the same configuration of subfaults as we used for the inversion of the strong ground-motion data from the Hyogo-ken Nanbu earthquake. We adopted the model proposed by Sekiguchi et al. (1996) on the basis of a minute location of the fault, which consists of three fault planes that we call subfault 1 (for Suma fault), subfault 2 (for Nojima), and subfault 3 (under Kobe), as shown in Figure 1. The source areas of the aftershocks used as empirical Green's functions are estimated from the corner frequencies to be $1 \times 1 \mathrm{~km}^{2}$. Then, in accordance with equations (1) and (3) and considering the parameters used by Kamae and Irikura (1998), we divided the three subfaults in $15 \times 21,21 \times$ 21 , and $24 \times 21$ elements. This is the basic element discretization model with 1260 unknown parameters to determine. Because the number of elements is very large we decided to merge nine elements to one to produce the superelement coarse grid model in which the number of parameters for the inversion is reduced from 1260 to 280 (140 $w_{i j}$ and $140 p t_{i j}$. Then, the weights for nine elements at each grid element of the superelement coarse grid model were assumed to be the same (see Fig. 4). A more drastic reduction was obtained when we used the ultraelement coarse grid model in which we merge 35 elements for subfault 1,49 elements for subfault 2, and 42 elements for subfault 3 , as shown in Figure 5. By using the ultraelements coarse grid model the number of inversion parameters reduces to 60 .

To test which of the grid-size models gives us the high-

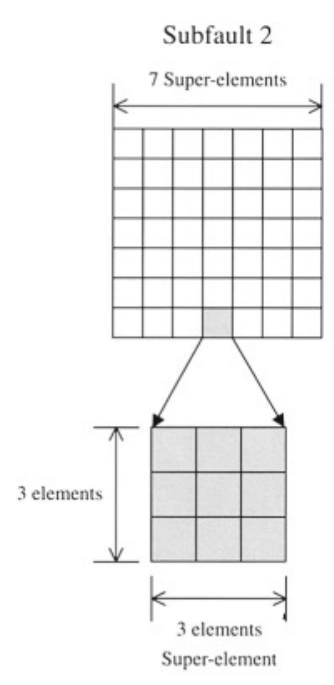

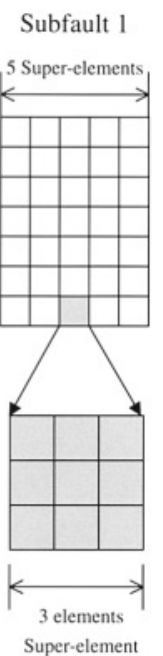

Super-element

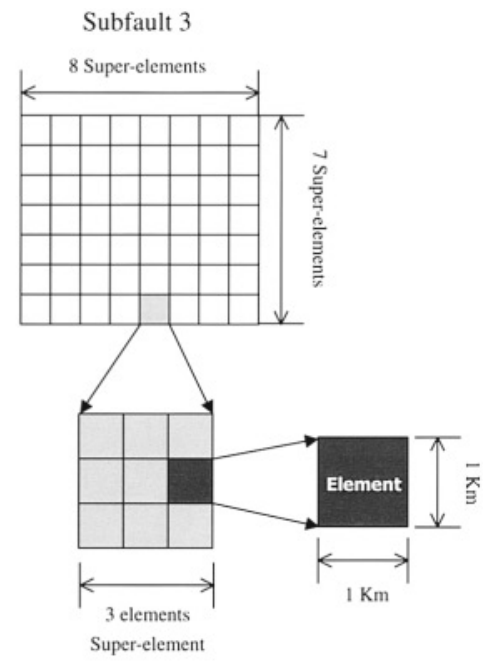

Figure 4. Superelement coarse gridding of the subfaults. 

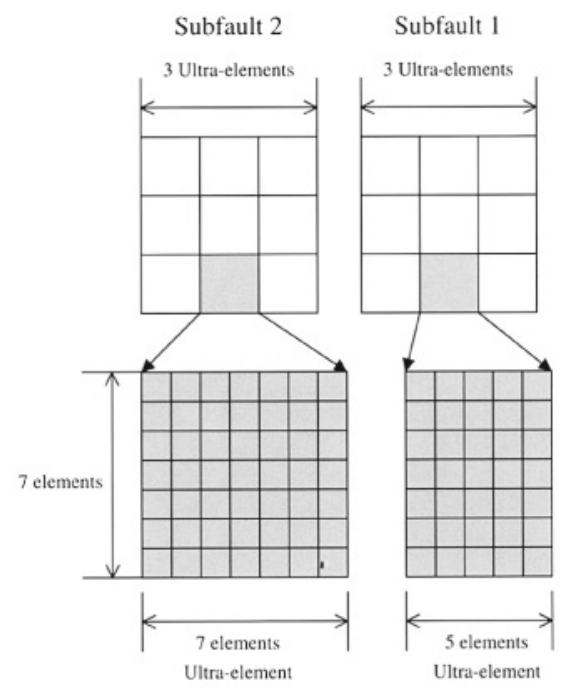

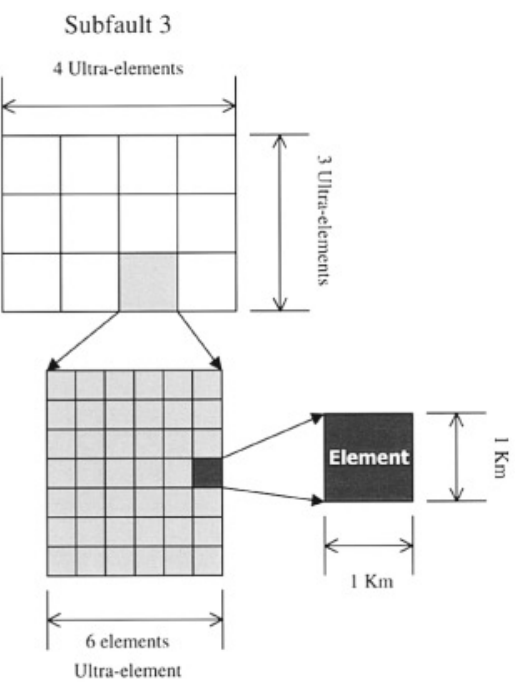

Figure 5. Ultraelement coarse gridding of the subfaults. est resolution with enough reliability we proposed two models of high-frequency radiation: models M5 and M6 shown in Figures 6 and 7, respectively. These models are used to calculate the forward problem. First, we calculate the strong motions, and second, the envelopes for all the stations using the weight and perturbation time distributions given by the models. Those envelopes were then used in the inversion problem to get the high-frequency radiation models that we

(a)

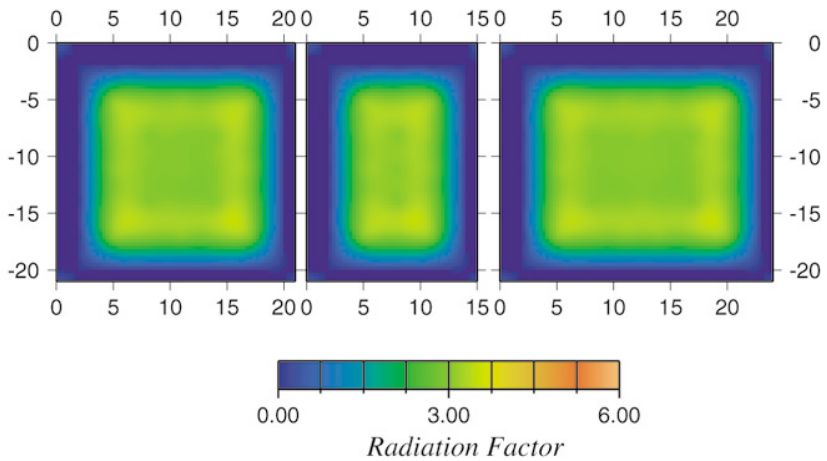

(b)
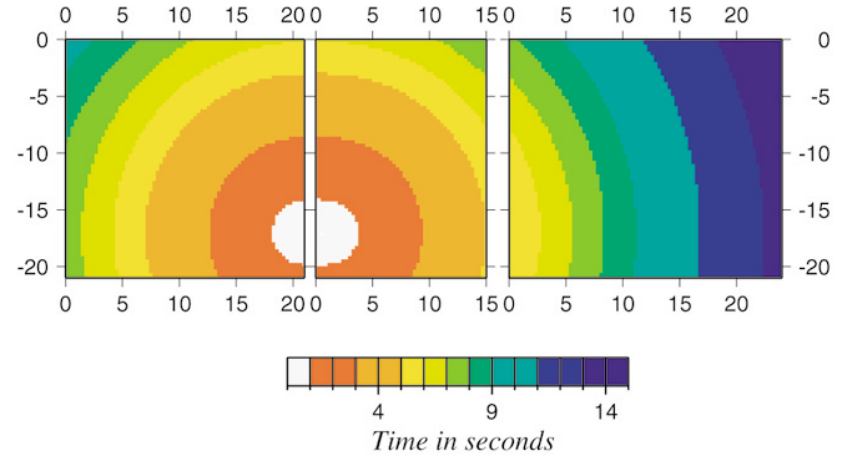

Figure 6. Model 5 (M5). (a) weight distribution; (b) rupture time progress. proposed, that is, M5 or M6. The proposed models of highfrequency radiation M5 and M6 were designed to be used by superelement and ultraelement coarse grid models, respectively; that is, to check specifically the resolution of each coarse grid model, so neither of them can be used for the other coarse grid model. From the physical point of view we are interested in detecting contrast of high-frequency radiation or delineating zones with and without radiation, so it

(a)

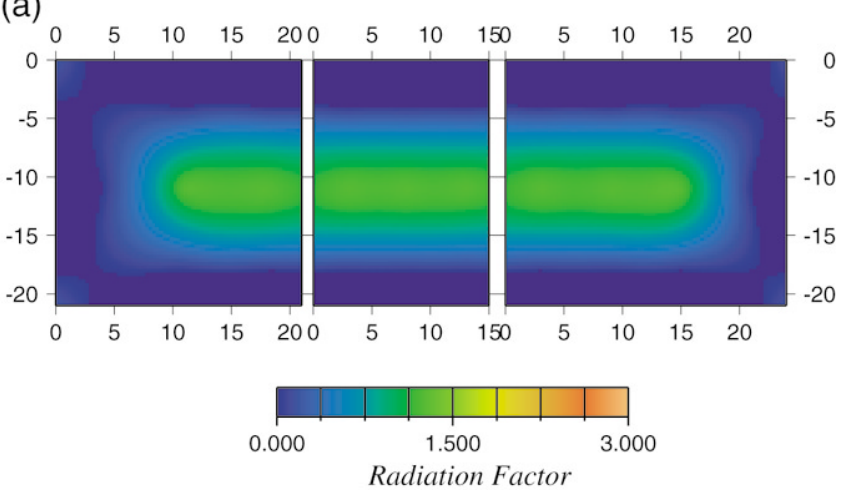

(b)

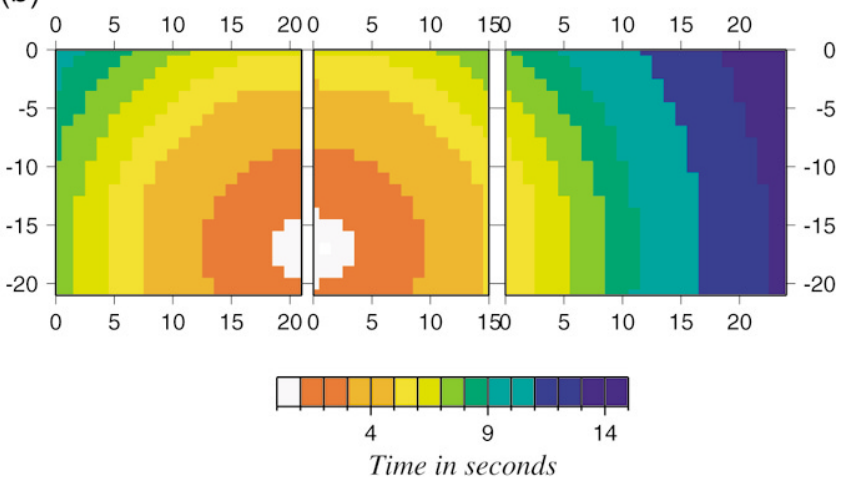

Figure 7. Model 6 (M6). (a) weight distribution; (b) rupture time progress. 
seems natural to use those models. We tested several combinations of discrete models and ranges of parameters: however, these two examples summarize the main results. Using the envelopes of the motions computed from the highfrequency radiation model M5 for the 16 stations we tried to invert them to recover the high-frequency radiation model M5. For this inversion, the superelement coarse grid model with 280 parameters was used. The resulting high-frequency radiation distribution and perturbation times from the inversion are shown in Figure 8. The good similarity of the original envelopes to those calculated with the inverted model are shown in the bottom of Figure 8. However, the distribution of high-frequency radiation given by the superelement coarse grid model discrete inversion was not accurate, and was very different from the proposed high-frequency radiation model M5 that was supposed to be obtained, despite the good envelope fit. This result implies that having a good envelope fit is not enough to guarantee that the distribution of the obtained high-frequency radiation is reliable.

Next, the ultraelement coarse grid model with 60 parameters was used. The envelopes of the motions computed from the high-frequency radiation model M6 for the 16 stations were used for the inverse problem. Figure 9 shows the high-frequency radiation distribution and perturbation times. The good similarity of the original envelopes to those calculated with the inverted model are shown in the bottom of Figure 9. Comparing the results shown in the top of Figure 9 with those from the proposed model M6 we can see that this inversion produces better performance, because the original high-frequency radiation model M6 is almost restored. In this case the accuracy of the inverted model reflects the accuracy of the envelope fitting. We conclude that this level of resolution is the one we can use for this set of data with enough reliability to perform the inversion. After checking the resolution we can apply the method to invert the distribution of the high-frequency radiation from the Hyogo-ken Nanbu earthquake.

\section{Application to 1995 Hyogo-ken Nanbu Earthquake}

The inversion was performed using the ultraelement coarse grid model, because it was found to be most reliable in the previous resolution test. We used the three-component acceleration data from the 16 stations depicted in Figure 2. Five aftershocks were used in the way that we indicated in Table 1.

\section{Results and Discussion}

The high-frequency radiation distribution inverted in the first trial is shown in Figure 10. The envelopes for the 16 stations are also shown in Figure 10, where the continuous line is for the observed envelopes and the dashed line is for the synthesized ones. Also, the reduction of the residuals with the increase of generation in the GA is shown in the bottom right side of Figure 10. In general, the fitting is acceptable for almost all the stations. This fitting could be improved if, for instance, the number of inversion parameters is increased (i.e., using the superelement coarse grid model), but the result would not be reliable, as shown by the resolution test. Instead of a perfect fitting with an unreliable inverted model, we have a fitting with some discrepancies that reflect our uncertainties in the inverted model, but higher reliability.

We tried the inversion several times using the ultraelement coarse grid model to check the influence of the radiation pattern. First, we corrected the radiation pattern for all the stations; second, we corrected it only for JMA stations; and third, the radiation pattern correction was done only for CEORKA stations. In others we tried only with horizontal components, or with the elimination of one or two stations. We judged each inversion on the basis of the residual value. We found that the best inversion (smallest residual) was obtained when all the stations and components were used, and when the radiation pattern was not corrected in all the stations. In fact Kamae et al. (1990) showed how the radiation pattern becomes uniform at frequencies higher than $2 \mathrm{~Hz}$.

Then, using all the stations without radiation pattern correction, we tried the inversion with the first population chosen randomly several times. The high-frequency radiation distribution for the best five inversions is plotted in Figure 11 with the residual values on the right side. The best model from the inversion is at the top. The main features of the high-frequency radiation obtained by the five inversions are very similar to each other and consistent with the results of the first inversion (Fig. 10). Considering that for all the inversions the high-frequency distribution model for the first generation of the GA was created randomly, the consistency of the five inverted models is proof of the stability of the solution.

We adopted the results of the inversion with the smallest residual as our final solution. These results are shown in Figure 12 along with the envelopes fitting and residual variation. As we can see in the top of Figure 12, the distribution of high-frequency radiation is concentrated in four zones, the largest one in subfault 1 , which extends from the bottom to the top of the fault; it is especially high close to the starting point. In subfault 2 , we found a high-radiation zone concentrated in a small area near the bottom. The other two highfrequency radiation zones are located in the middle and top of subfault 3. All four zones are well correlated with the high-speed zones. In general, the high-frequency motion is produced when the rupture starts simultaneously from a line or it abruptly changes its propagation velocity (Madariaga, 1977; Boatwright, 1982). In our case the zone located in subfault 1 seems to be related to either a step-over of the rupture (proposed by Kakehi et al., 1996) from the initial fault plane to the other fault plane of subfault 2 , or to the initiation of the rupture itself. The high-frequency concentration zone located in the top of the subfault 3 may be an indicator of bifurcation of the fault into two segments (Sekiguchi et al., 2000). In Figure 1 we can observe that around 


\section{High frequency radiation}

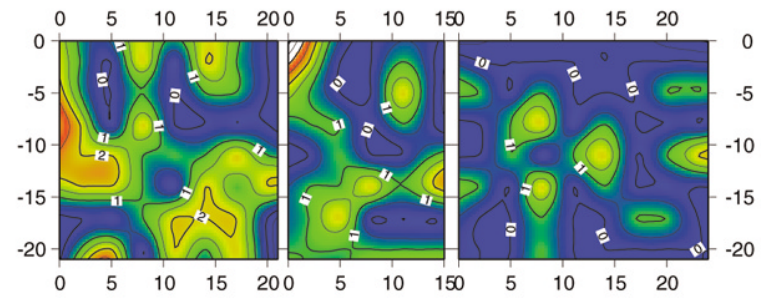

\section{Rupture time History}

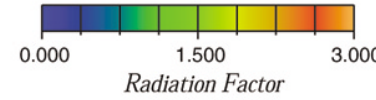

\section{Perturbation times distribution}
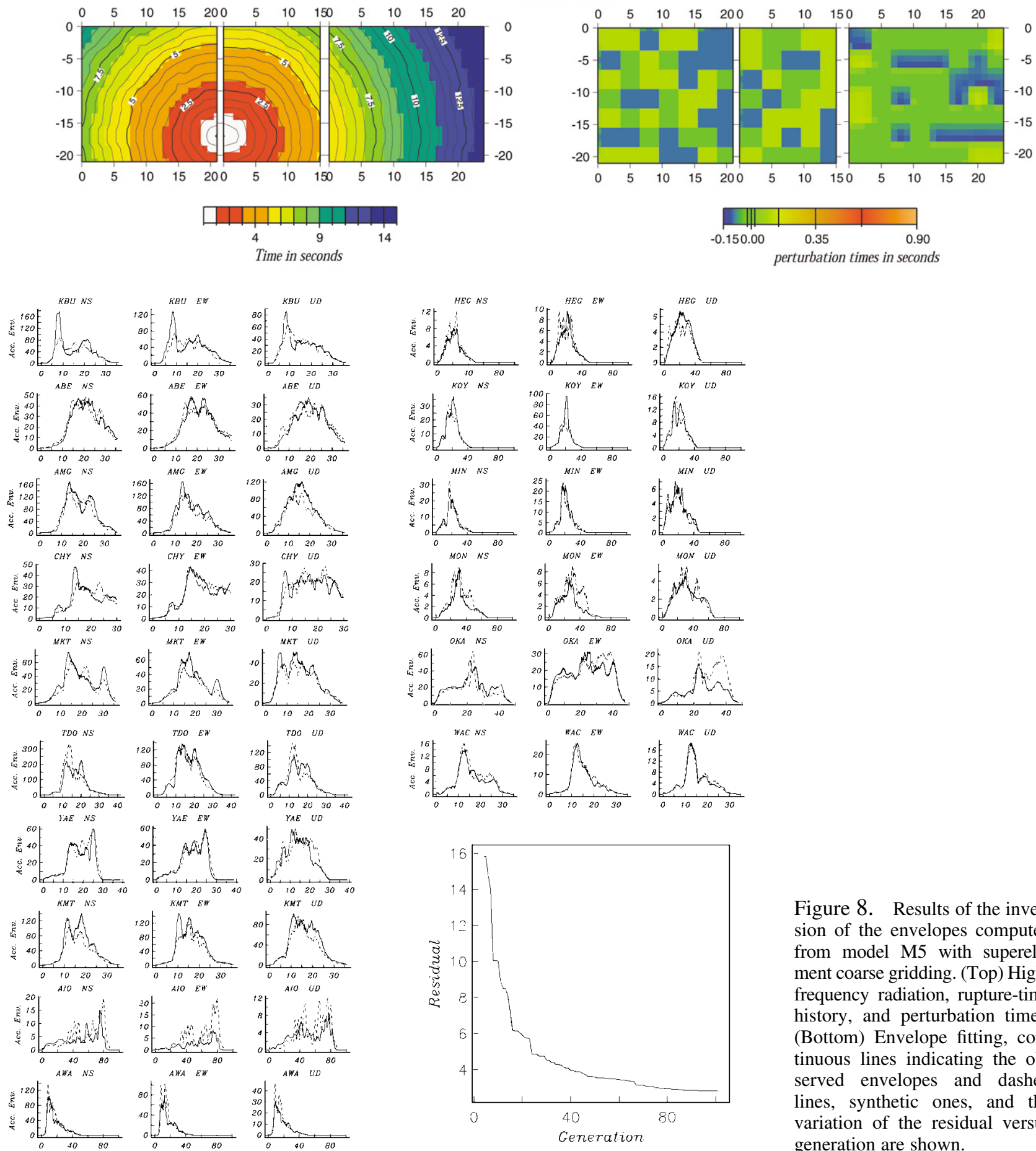

Figure 8. Results of the inversion of the envelopes computed from model M5 with superelement coarse gridding. (Top) Highfrequency radiation, rupture-time history, and perturbation times. (Bottom) Envelope fitting, continuous lines indicating the observed envelopes and dashed lines, synthetic ones, and the variation of the residual versus generation are shown. 


\section{High frequency radiation}

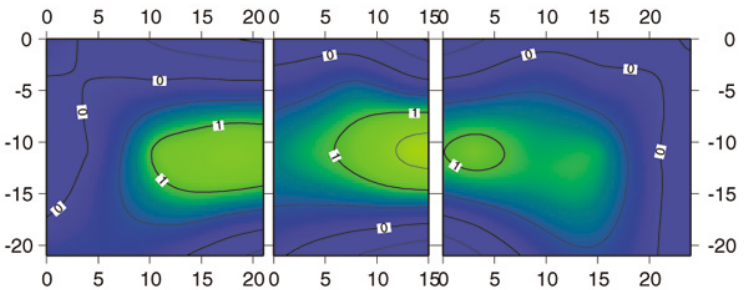

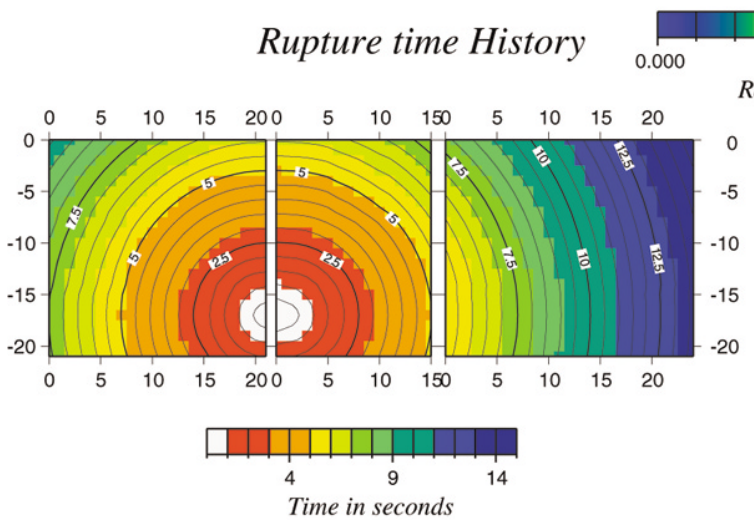

Figure 9. Results of the inversion of the envelopes computed from model M6 with ultraelement coarse gridding. (Top) Highfrequency radiation, rupture-time history, and perturbation times. (Bottom) Envelope fitting, continuous lines indicating the observed envelopes and dashed lines, synthetic ones; and the variation of the residual versus generation are shown.
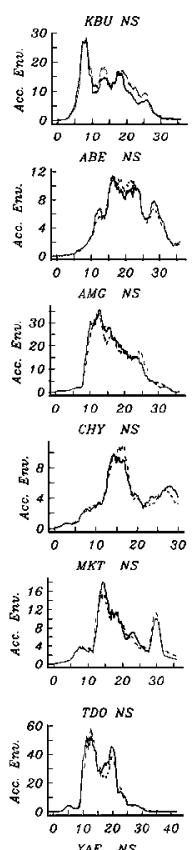

12 YAE NS
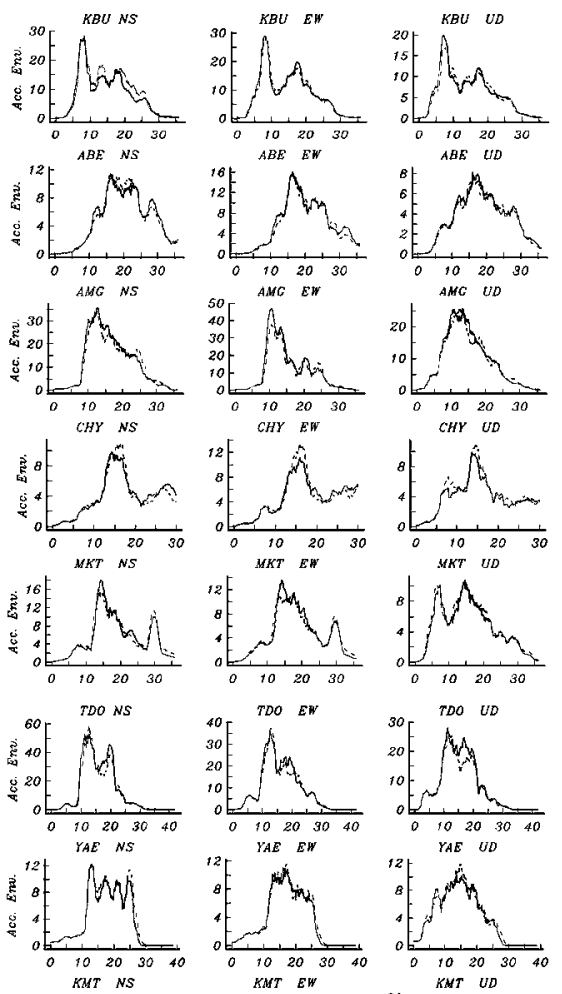

$A M C$ UD
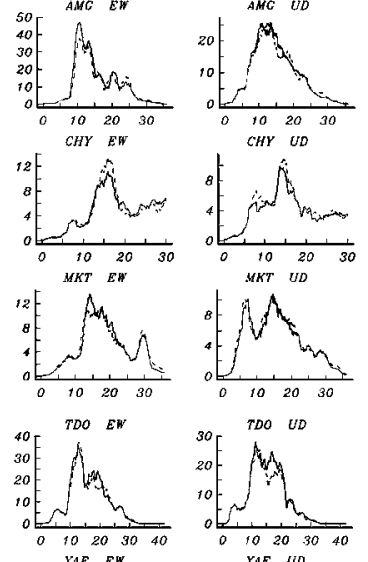

30 TDO UD
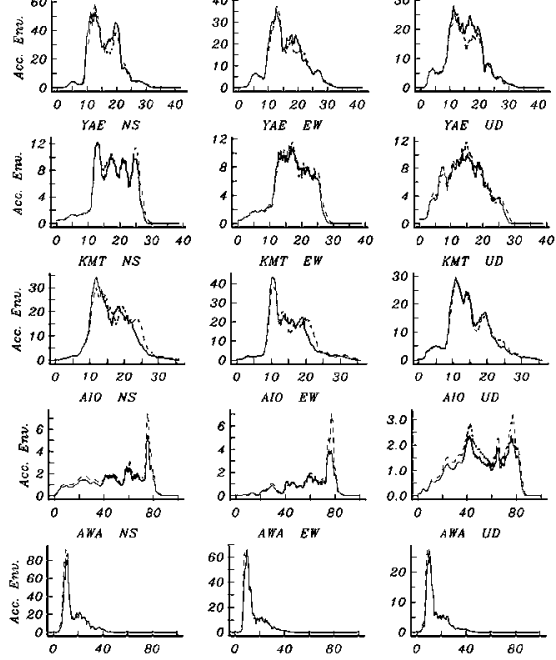

Perturbation times distribution

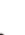

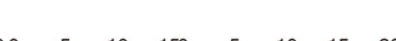

$n$ 


\section{High frequency radiation}

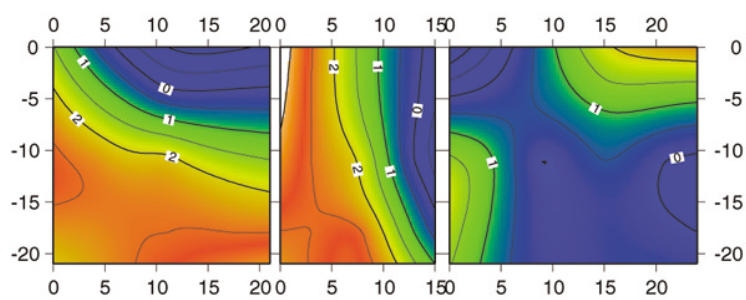

\section{Rupture time History $\quad 0.000 \quad \begin{gathered}1.500 \\ \text { Radiation Factor }\end{gathered}{ }^{3.000} \quad$ Perturbation times distribution}
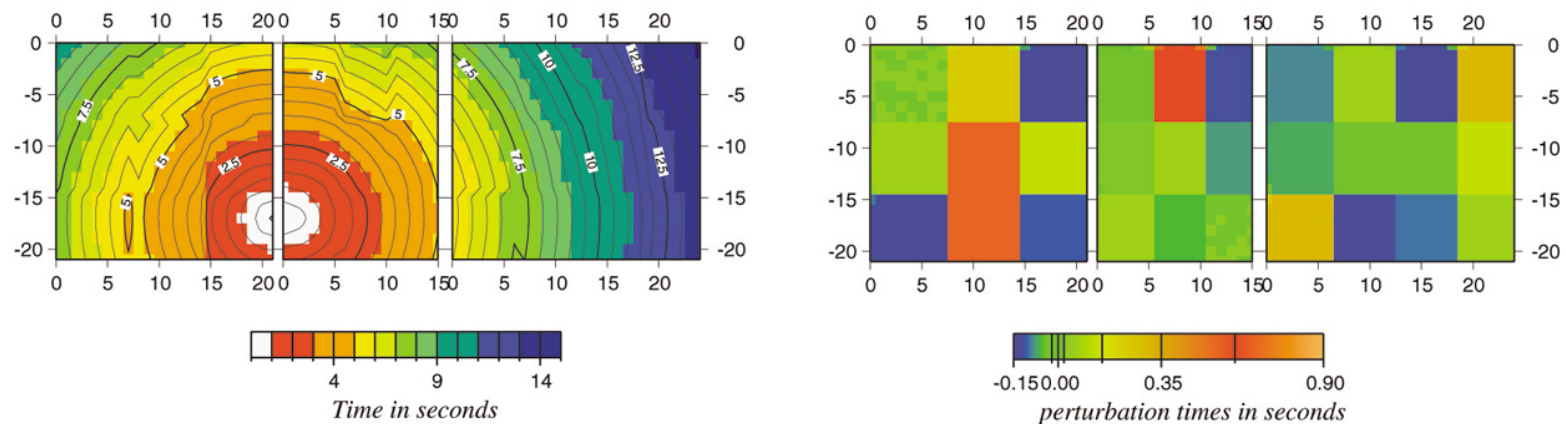
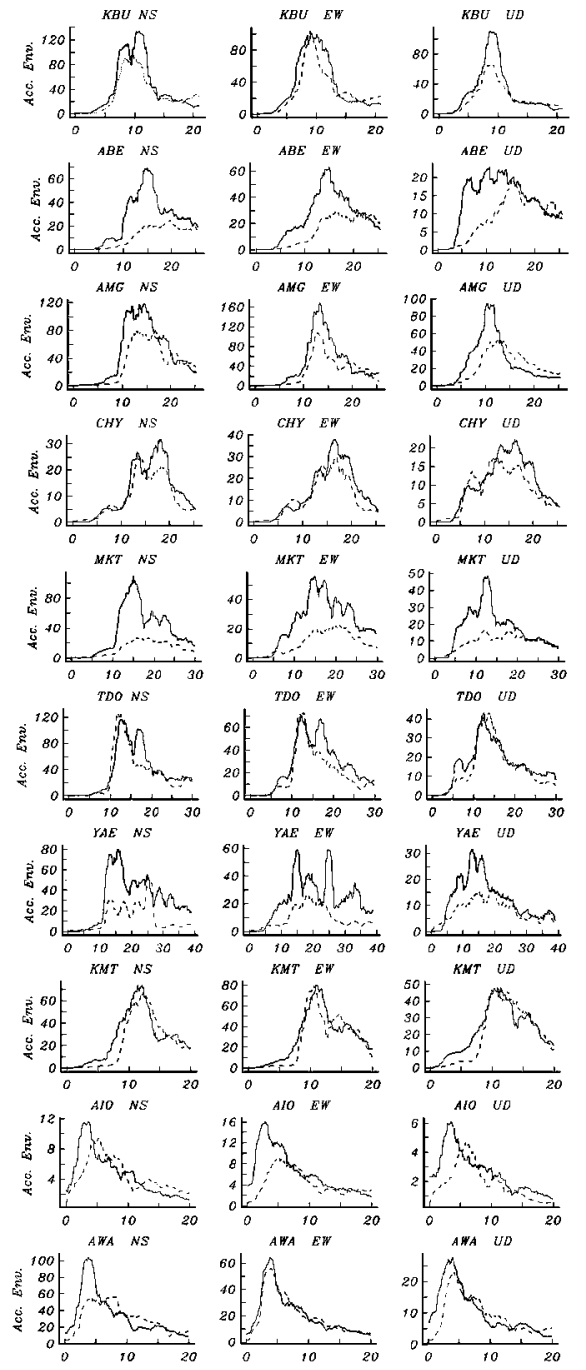
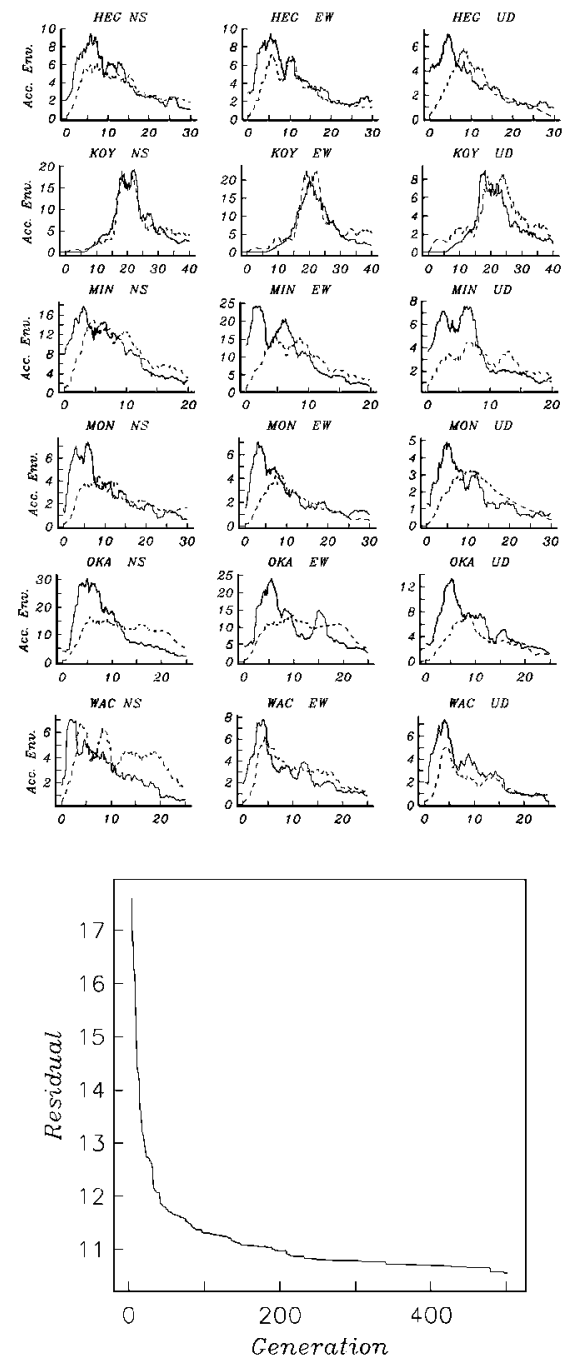

Figure 10. Results of the inversion of the 1995 Hyogo-ken Nanbu earthquake with ultraelement coarse gridding. (Top) Highfrequency radiation, rupture-time history, and perturbation times. (Bottom) Envelope fitting, continuous lines indicating the observed envelopes and dashed lines, synthetic ones; and the variation of the residual versus generation are shown. 

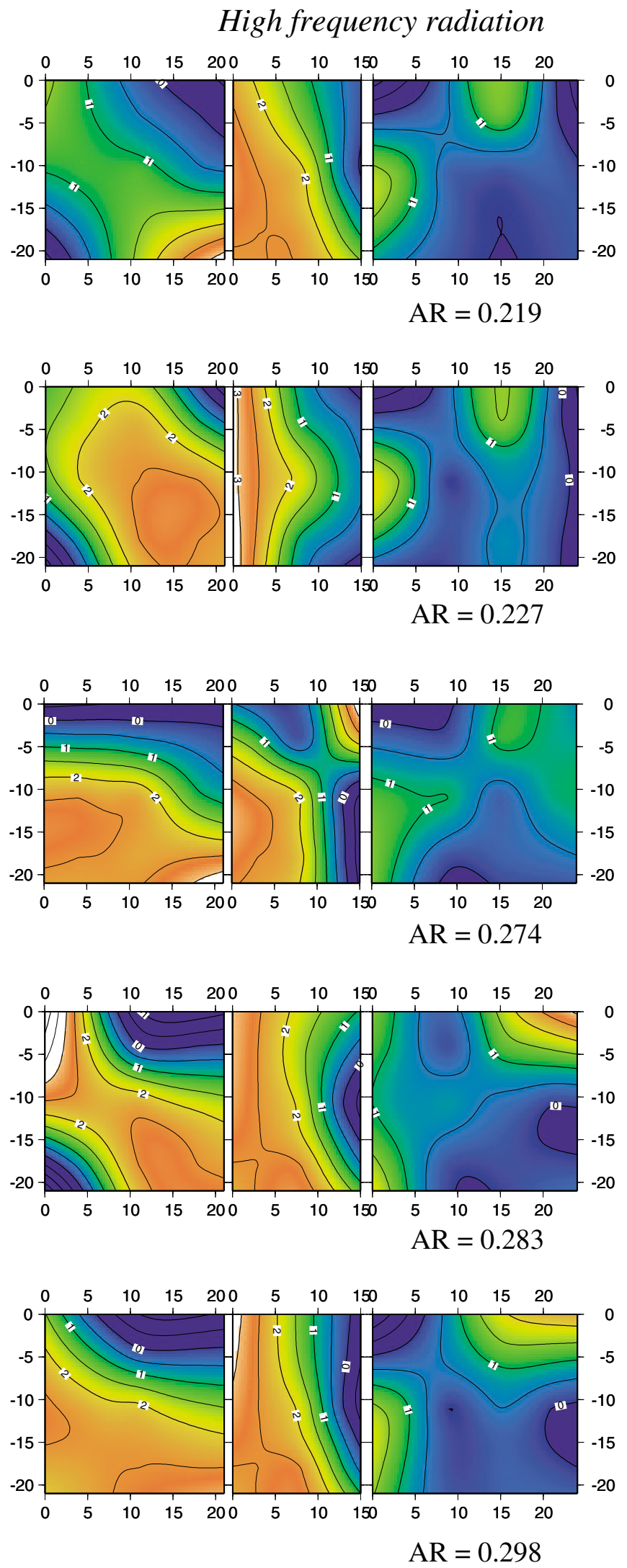

Figure 11. High-frequency radiation distributions obtained from several trials. the middle of subfault 3 the traces of geological faults are branched into two, one in the northern part that is the continuation of the Suwayama fault called Gosukebashi, and the other in the south called the Ashiya fault. Yoshida et al. (1996), in a joint inversion of geodesic data and seismic waveform data, analyzed the rupture process of these faults but they could not determine which one of the faults ruptured. However, the rupture of the Ashiya fault is actually clearly demonstrated by Sekiguchi et al. (2000).

The common features of many low-frequency inversions done for this earthquake (Horikawa et al., 1996; Ide et al., 1996; Sekiguchi et al., 1996; Wald, 1996; and Yoshida et al., 1996) show that the slip in subfault 2 was very large near the surface. The high-frequency radiation zone found here is the complement of the large slip zone of the lowfrequency inversion, marking off the lower limit of the asperity.

Our result has some features similar to those obtained by Kakehi et al. (1996), for example, the points denoted as $\mathrm{B}$ and $\mathrm{C}$ by these authors are well correlated with the band of radiation that we found in subfault 1. A similar band can be observed in the distribution of dynamic-static friction coefficients estimated by Bouchon et al. (1998). Also, the highfrequency radiation zone found in the top of subfault 3 is similar to that denoted as D in Kakehi et al. (1996).

However, there are also some discrepancies of our results with those obtained by Kakehi et al. (1996). These discrepancies are natural because the model of Kakehi et al. is different from the model that we use. They assumed only one fault plane and used different stations and different aftershock records as empirical Green's functions. These authors used a coarse grid size similar to our ultraelement coarse grid size. In our simulation we did not find any concentration of high frequency at the points called A and E in Kakehi et al. (1996).

Both the results of Kakehi et al. (1996) and our results show clearly that the high-frequency radiation distributed in the fault plane is different from the slip distribution obtained by the inversion in low frequencies. Then, the high-frequency radiation distribution should be included in the forward modeling of the distribution of peak ground velocities to explain appropriately the damage distribution, especially when structures with periods shorter than $1 \mathrm{sec}$ are included.

\section{Conclusions}

We inverted the high-frequency radiation of the 1995 Hyogo-ken Nanbu earthquake. We found that the reliability of the high-frequency envelope inversion is influenced by the number of unknown parameters, which depends on the grid size. First, we examined the validity of the inversion using models with different grid sizes, and found that the ultraelement coarse grid model with 60 parameters had the higher resolution, compared with the superelements coarse grid model with 280 parameters. The resolution test that we conducted cannot be generalized because it depends on the 


\section{High frequency radiation}

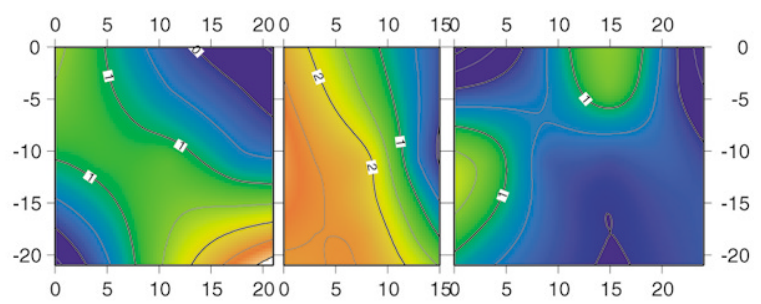

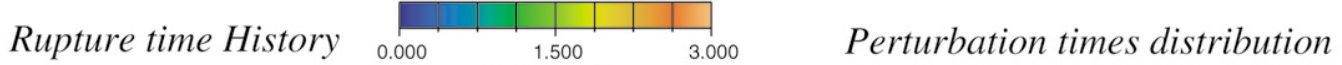

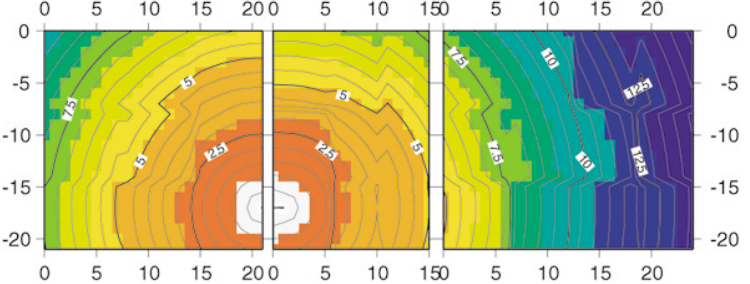
Radiation Factor
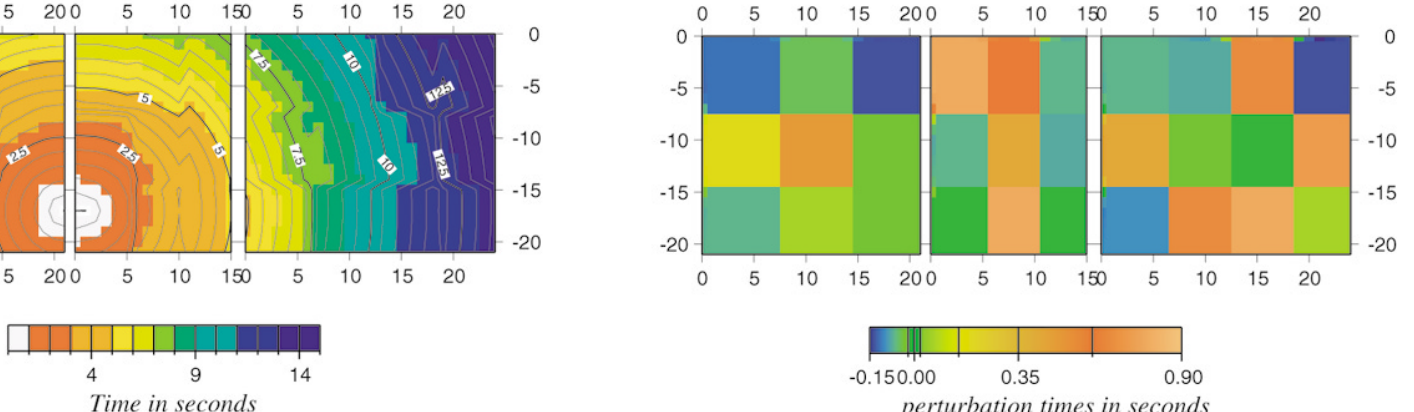

perturbation times in seconds
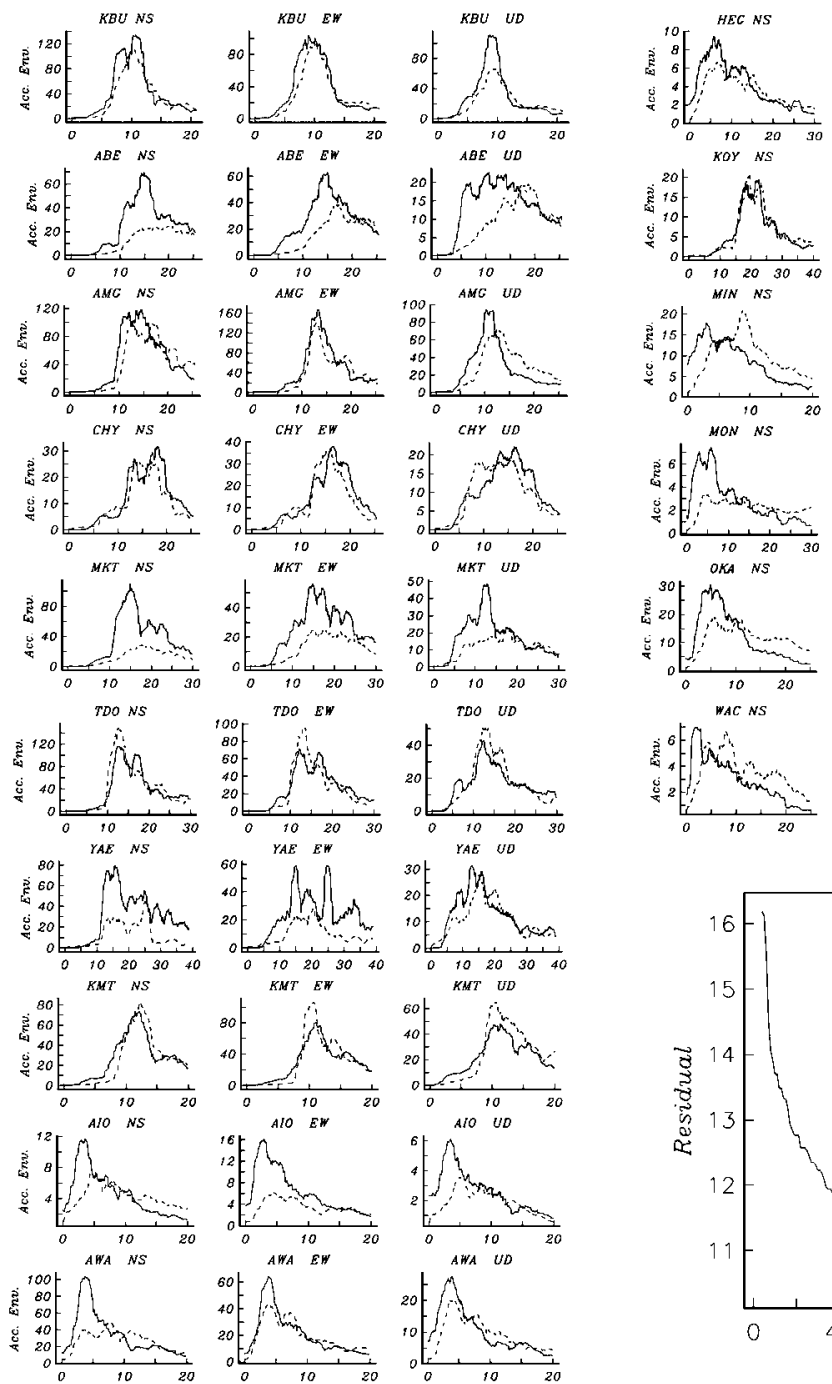
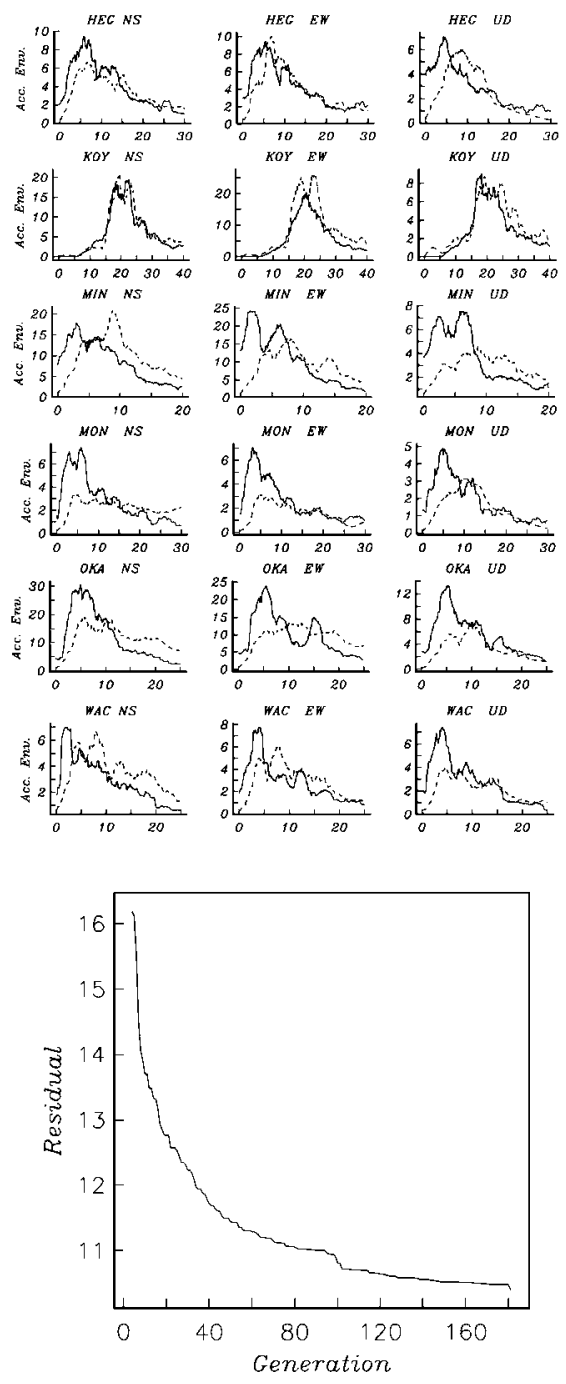

Figure 12. Results of the inversion of the 1995 Hyogo-ken Nanbu earthquake, for the best model. (Top) High-frequency radiation, rupture-time history, and perturbation times. (Bottom) Envelope fitting, continuous lines indicating the observed envelopes and dashed lines, synthetic ones; and the variation of the residual versus generation are shown. 
configuration of the fault model and the array of stations. However, we believe that a relation between the number of parameters inverted and the number of stations exists. Therefore, similar testing has to be done before performing the inversion for each case. Second, we performed the envelope inversion for high-frequency radiation using the ultraelement coarse grid model with 60 parameters. We obtained the best fit when the radiation pattern was not corrected. We adopted the results of the inversion with the smallest residual as our final solution. In our final solution we found that the distribution of high-frequency radiation is concentrated in four zones. The zone located in subfault 1 , corresponded to the starting of the rupture and a step-over from subfault 1 to the Nojima fault. The high-frequency radiation zone located in subfault 2 was found to coincide with the lower limit of the slip inverted from low frequencies by many authors. The high-frequency zone in the top of subfault 3 coincides with the point of bifurcation into two fault planes of Sekiguchi et al. (2000).

We conclude that the procedure applied in this article successfully inverts the high-frequency radiation. Also, we have succeeded in determining the most reliable model for the inversion through the resolution test in which it was found that the resolution depends of the number of the stations. Another finding was that the inversion does not require of the correction for radiation pattern.

\section{Acknowledgments}

We thank the organizations who provided the recordings: Committee of the Earthquake Observation and Research in the Kansai Area (CEORKA), and Japan Meteorological Agency (JMA). We thank Prof. Gustavo Ayala who helped us to improve substantially the manuscript. This research was partially supported by Conacyt under Grant J27740T/5722. Most of the figures were created using Generic Mapping Tools software (Wessel and Smith, 1998).

\section{References}

Boatwright, J. (1982). A dynamic model of far-field acceleration, Bull. Seism. Soc. Am. 72, 1049-1068.

Bouchon, M., H. Sekiguchi, K. Irikura, and T. Iwata (1998). Some characteristics of the stress field of the 1995 Hyogo-ken Nanbu (Kobe) earthquake, J. Geophys. Res. 103, 24271-24282.

Cocco, M., and J. Boatwright (1993). The envelopes of acceleration time histories, Bull. Seism. Soc. Am. 83, 1095-1114.

Dietrich, J. (1979). Modeling of rock friction: experimental results and constitutive equations, J. Geophys. Res., 84, 2161-2168.

Fukuyama, E., and T. Mikumo (1993). Dynamic rupture analysis: inversion for the source process of the 1990 Izu-Oshima, Japan, earthquake $(\mathrm{M}=6.5)$, J. Geophys. Res. 98, 6529-6542.

Gusev, A. A., and V. M. Pavlov (1991). Deconvolution of squared velocity waveform as applied to study of a noncoherent short-period radiator in the earthquake source, Pure Appl. Geophys. 136, 235-244.

Holland, J. H. (1975). Adaptation in Natural and Artificial Systems, MIT Press, Cambridge, Massachusetts.
Horikawa, H., K. Hirahara, Y. Umeda, M. Hashimoto, and F. Kusano (1996). Simultaneous inversion of gedetic and strong-motion data for the source process of the Hyogo-ken Nanbu, Japan, earthquake, J. Phys. Earth 44, 455-471.

Ide, S., M. Takeo, and Y. Yoshida (1996). Source model of the 1995 Hyogo-ken earthquake determined by near-field strong-motion records, J. Phys. Earth 44, 649-653.

Irikura, K. (1986). Prediction of strong acceleration motion using empirical Green's function, Proc. 7th Jpn. Earthquake Eng. 8, 37-42.

Kakehi, Y., and K. Irikura (1996). Estimation of high frequency wave radiation areas on the fault plane by the envelope inversion of acceleration seismograms, Geophys. J. Int. 125, 892-900.

Kakehi, Y., K. Irikura, and M. Hoshiba (1996). Estimation of high frequency wave radiation areas on the fault plane of the 1995 Hyogoken Nanbu earthquake by the envelope inversion of acceleration seismograms, J. Phys. Earth 44, 505-517.

Kamae, K., and K. Irikura (1998). Source model of the 1995 Hyogo-ken Nanbu earthquake and simulation of near-source ground motion, Bull. Seism. Soc. Am. 88, 400-412.

Kamae, K., K. Irikura, and Y. Fukuchi (1990). Prediction of strong ground motion for M 7 earthquake using regional scaling relations of source parameters (In Japanese with English abstract), J. Struct. Construct. Eng. Architectural Institute of Japan 416, 57-70.

Levine, D. (1996). Users guide to the PGApack parallel genetic algorithm library, Internal report Argone National Laboratory, ANL-95/18.

Madariaga, R. (1977). High-frequency radiation from crack (stress drop) model of earthquake faulting, Geophys. J. R. Astr. Soc. 51, 625-651.

Rice, J. R., and A. L. Ruina (1983). Stability of steady frictional slipping, J. Appl. Mech. 105, 343-349.

Sekiguchi, H., K. Irikura, T. Iwata, Y. Kakehi, and M. Hoshiba (1996). Minute location of faulting beneath Kobe and the waveform inversion of the source process during the 1995 Hyogo-ken Nanbu, Japan, earthquake using strong ground motion records, J. Phys. Earth 44, 473487.

Sekiguchi, H., K. Irikura, and T. Iwata (2000). Fault geometry at the rupture termination of the 1995 Hyogo-ken Nanbu earthquake, Bull. Seism. Soc. Am. 90, 117-133.

Wald, D. J. (1996). Slip history of the 1995 Kobe, Japan, earthquake determined from strong motion, telesismic, and geodetic data, J. Phys. Earth 44, 489-503.

Wessel, P., and W. H. F. Smith (1998). New improved version of Generic Mapping Tools released, EOS 79, 579.

Yoshida, S., K. Koketsu, B. Shibazaki, T. Sagiya, T. Kato, and Y. Yoshida (1996). Joint inversion of near- and far-field waveforms and geodetic data for the rupture process of the 1995 Kobe earthquake, J. Phys. Earth 44, 437-454.

Zeng, Y., L. Aki, and T.-L. Teng (1993). Mapping of the high frequency source radiation for the Loma Prieta earthquake, California, J. Geophys. Res. 98, 11,981-11,993.

Instituto de Ingeniería, UNAM

Ciudad Universitaria, Apdo. 70-472

Coyoacán 04510, México, D. F., Mexico

joagg@pumas.iingen.unam.mx

(J.A.)

Disaster Prevention Research Institute

Kyoto University

Uji 611, Japan

irikura@egmdpri01.dpri.kyoto-u.ac.jp

(K.I.)

Manuscript received 18 June 2002. 